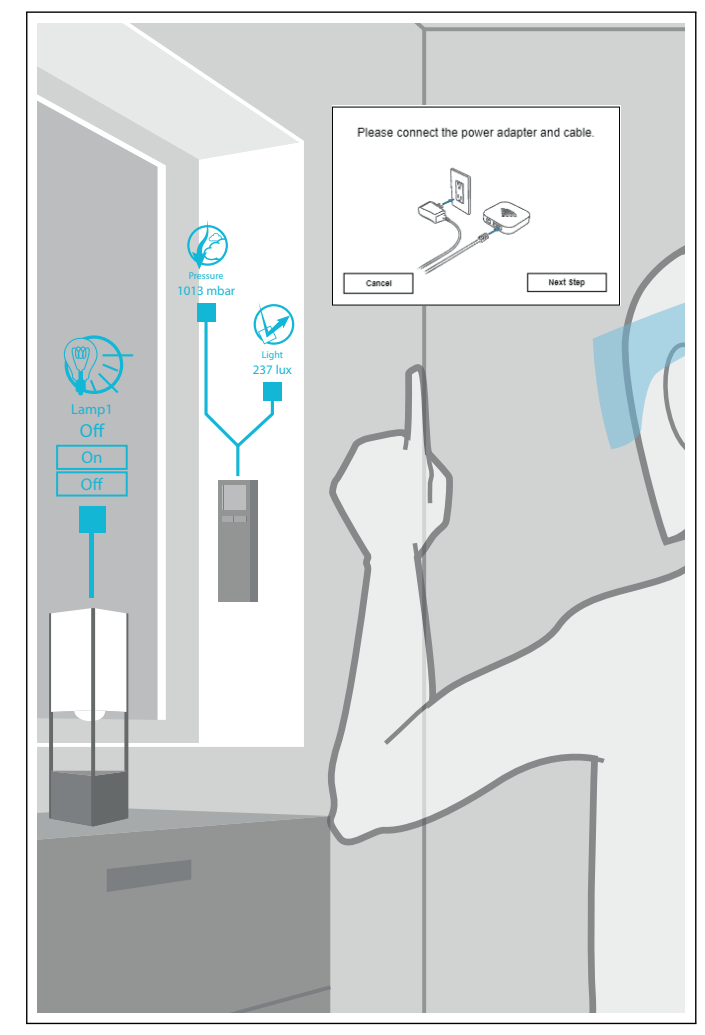

Figure 1: Setup Wizard for Smart Devices in Mixed Reality.

\title{
Workflow-based Setup of Smart Devices in Mixed Reality
}

\author{
Ronny Seiger \\ University of St.Gallen \\ St.Gallen, Switzerland \\ ronny.seiger@unisg.ch
}

\author{
André Kühnert \\ Uwe Aßmann \\ Technische Universität Dresden \\ Dresden, Germany \\ andre.kuehnert@tu-dresden.de \\ uwe.assmann@tu-dresden.de
}

\section{ABSTRACT}

New types of smart devices are emerging every day providing end-users with new ways of interacting with the loT. Setup and configuration of these devices are, however, rather complex and require technical expertise. Especially in smart homes users often lack these skills and need assistance with setting up devices, which makes them hesitant to adopt new technologies. We present a mixed reality application to support users with the setup of loT devices. The setup tasks are modelled and executed as formal interactive workflows. An intuitive wizard guides users and presents additional task-related multimedia information in mixed reality. A semantically enriched loT middleware provides data and control of the loT devices. We conducted a brief user study to evaluate the application's usability.
\end{abstract}

\section{CCS CONCEPTS}

- Human-centered computing $\rightarrow$ Mixed / augmented reality; Ubiquitous and mobile computing systems and tools; • Applied computing $\rightarrow$ Business process management systems.

IoT 2019, October 22-25, 2019, Bilbao, Spain

( 2019 Copyright held by the owner/author(s).

This is the author's version of the work. It is posted here for your personal use. Not for redistribution. The definitive Version of Record was published in 9th International Conference on the Internet of Things (IoT 2019), October 22-25, 2019, Bilbao, Spain, https://doi.org/10.1145/3365871.3365909. 


\section{KEYWORDS}

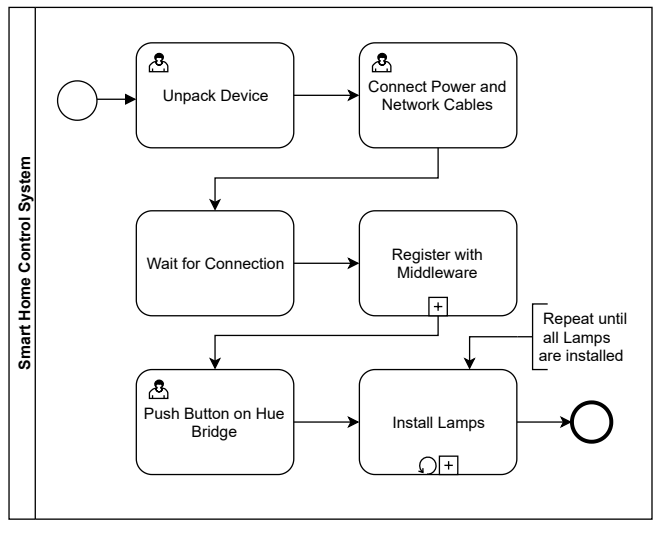

Figure 2: Installation Workflow for Hue Lamps from Philips.

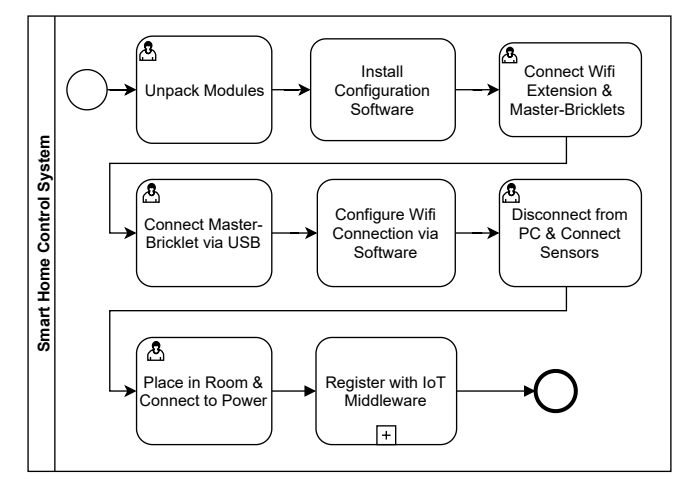

Figure 3: Installation Workflow for Sensors from Tinkerforge.

${ }^{1}$ https://www.meethue.com

${ }^{2}$ https://www.tinkerforge.com

${ }^{3}$ https://www.openhab.org/
Mixed Reality; Smart Device Setup; IoT Workflows; Smart Home

\section{ACM Reference Format:}

Ronny Seiger and André Kühnert, Uwe Aßmann. 2019. Workflow-based Setup of Smart Devices in Mixed Reality. In 9th International Conference on the Internet of Things (IoT 2019), October 22-25, 2019, Bilbao, Spain. ACM, New York, NY, USA, 4 pages. https://doi.org/10.1145/3365871.3365909

\section{INTRODUCTION}

Installation and configuration of loT devices is often complex and requires technical/expert knowledge. In non-expert domains such as smart homes users are keen to interact with their loT surroundings and devices. However the complexity of installing new loT devices makes end-users reluctant of adapting these technologies [1]. While related work is mainly focussing on direct device and user interactions $[1,3,10]$ or automating the integration and configuration aspects using agents $[4,5,9]$, we present a user-friendly mixed reality (MR) app to guide end-users with the setup of loT devices based on formal flexible and interactive workflows augmented with a configuration wizard (cf. Fig. 1).

\section{SMART HOME USE CASES}

In smart homes users-mostly non-experts-are confronted with a wide spectrum of smart consumer devices with very specific installation procedures often requiring printed or digital installation manuals and even Internet lookups. We choose two examples to illustrate the complexity of these processes and the need for an application assisting end-users. Fig. 2 shows the simplified workflow of installing a new Philips Hue ${ }^{1}$ light system. Fig. 3 presents a more complex setup workflow of room climate monitoring sensors from Tinkerforge ${ }^{2}$. Both processes consist of manual tasks that have to be accompanied by text instructions, images and videos to aid users; and of software parts to be automated.

\section{SMART DEVICE SETUP IN MIXED REALITY}

\section{System Overview}

Fig. 4 shows an overview of the system architecture. The MR app interacts as a client application with the IoT platform containing a workflow management system (WfMS), a middleware (openHAB ${ }^{3}$ ) extended by a semantic access layer and a knowledge base with semantic data about the loT devices.

\section{Optical Device Recognition}

An important feature of the app to have is the ability to identify the individual loT devices. We evaluated various open-source solutions for optical object recognition. Smart homes are subject to changing context and lighting conditions and furnished very individually. Many objects and loT devices 


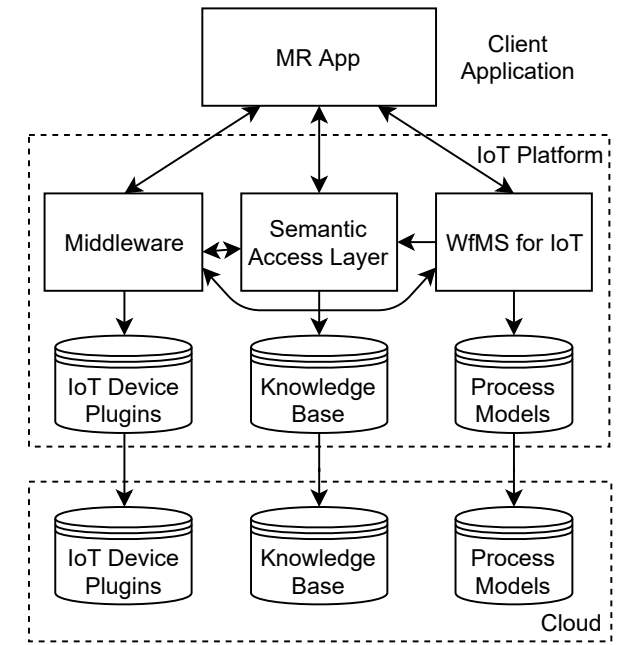

Figure 4: Overall System Architecture.
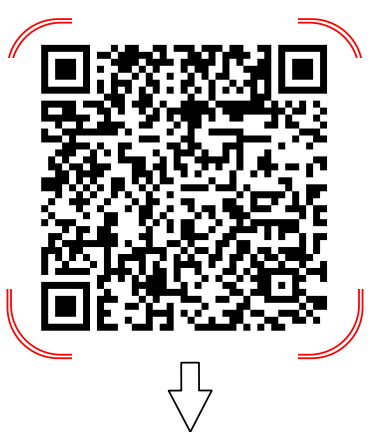

Bld: Thing-Actuator-Philips_Hue Devld: Thing-Actuator-Philips_Hue_Iris2 Wfld: Workflow-Actuator-Philips_Hue

Figure 5: QR Code Scan for Identifying a Smart Device and Setup Workflow. are hard to detect and distinguish in varying positions and from different angles. All investigated libraries proved rather unreliable for these settings. Thus we decided to use QR codes on the devices or packaging as a flexible and reliable means for object (instance) identification (cf. Fig. 5). Here we encode IDs of the device (Devld), of the setup workflow (Wfld), and of the middleware plugin (Bld).

\section{IoT Middleware \& Semantic Device Data}

The IoT middleware is used for integrating the heterogeneous set of loT devices via specific plugins and providing a unified service-based interface to access the devices' functionalities. The Semantic Access Layer is an extension for the middleware to include and store an ontological description of the IoT devices, their capabilities and context based on DogOnt in local or remote knowledge bases [2].

\section{Automated Configuration Workflows}

The individual setup tasks are modelled as workflows in a BPMN-like language [8]. Here workflows facilitate the integration of automatic tasks to call services of the middleware and external applications and of manual tasks with textual descriptions, pictures or videos referenced via local or remote URIs to be displayed during execution of the individual tasks. Fig. 6 depicts a particular manual task and the associated instructions. We use a specific WfMS for loT to execute the workflows providing means for tracing and analysing the workflow execution and presenting context-specific instructions [7].

\section{Mixed Reality Device Configuration}

Upon its start the MR app presents users with options for setting up new devices or for exploring/controlling existing devices. After entering the Setup mode, the app waits for a QR code to be present (cf. Fig. 5). The extracted workflow ID is used to find the device class's setup workflow from a repository, which is instantiated by the WfMS. Holograms are displayed based on information from the manual tasks contained in the workflow model (cf. Fig. 7). The user is able to go through the workflow steps and to monitor the execution in the WfMS's visualizer. Non-manual tasks (e. g., to register a device with the middleware) are executed automatically. Once the workflow is executed a hologram for the device based on the semantic information is generated and can be placed at the device's physical location (cf. Fig. 1) to be interacted with in MR (e.g., via the HoloFlows app [6]).

\section{EVALUATION \& DISCUSSION}

We conducted a preliminary user study with $5(\mathrm{PhD})$ students from IT. The participants had to setup a Philips Hue lamp and two Tinkerforge sensors following the previously presented workflows and being supported by the wizard in the MR app. Despite a lack of experience with loT devices, all users were able to follow the instructions and setup the new devices. It took them approx. 5 minutes on average per device. All users found the app useful and supportive as detailed and interactive instructions were 


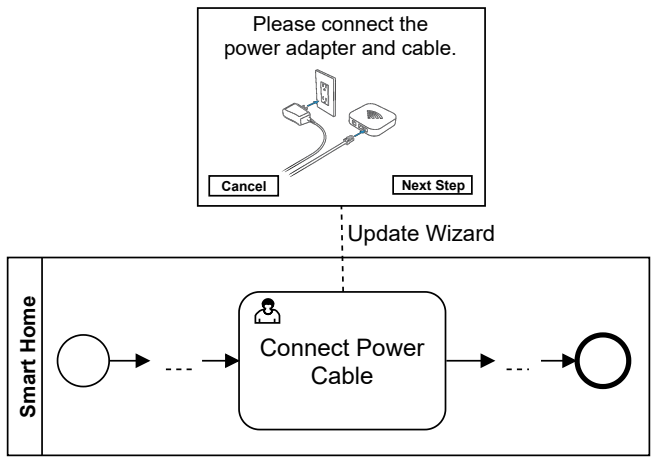

Figure 6: A Manual Workflow Task and Associated Instructions of Wizard.

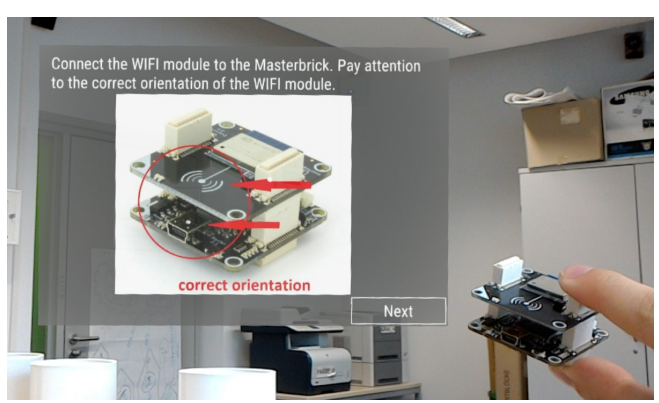

Figure 7: Live View of a Configuration Task in Mixed Reality. displayed at the right points in time and hands were free for device setup. Manual steps (e. g., reading user manuals and adding devices to the middleware) could be saved due the additional information and automatic service calls in the underlying workflows. The MR app can be used in various contexts to guide users through manifold assembly, setup and configuration processes. The workflows and semantic models have to be provided by manufacturers or other domain experts. The loT platform and MR app can then be used to load the workflows and data, instruct users and setup new loT devices.

\section{CONCLUSION}

We presented a mixed reality application to guide end-users with the setup and configuration of new loT devices in the context of smart homes. The underlying loT platform supports flexible setup workflows in a generic way such that the MR app can also be used for assisting users in other IoT application domains, which will be part of our future work. The individual workflow tasks and physical devices/world can be augmented with different types of multimedia content facilitating the completion of the manual activities by the users. All in all, the MR app shows potential for supporting all kinds of assembly and configuration workflows for manual and automated tasks and loT devices.

\section{REFERENCES}

[1] Adrian A de Freitas, Michael Nebeling, Xiang 'Anthony' Chen, Junrui Yang, Akshaye Shreenithi Kirupa Karthikeyan Ranithangam, and Anind K Dey. 2016. Snap-to-it: A user-inspired platform for opportunistic device interactions. In Proceedings of the 2016 CHI Conference on Human Factors in Computing Systems. ACM, 5909-5920.

[2] Steffen Huber, Ronny Seiger, André Kühnert, Vasileios Theodorou, and Thomas Schlegel. 2016. Goal-based semantic queries for dynamic processes in the internet of things. International Journal of Semantic Computing 10, 02 (2016), 269-293.

[3] Fahim Kawsar, Tatsuo Nakajima, and Kaori Fujinami. 2008. Deploy spontaneously: supporting end-users in building and enhancing a smart home. In Proceedings of the 10th international conference on Ubiquitous computing. ACM, 282-291.

[4] Simon Mayer, Nadine Inhelder, Ruben Verborgh, Rik Van de Walle, and Friedemann Mattern. 2014. Configuration of smart environments made simple: Combining visual modeling with semantic metadata and reasoning. In 2014 International Conference on the Internet of Things (IOT). leee, 61-66.

[5] Simon Mayer, Markus Schalch, Marian George, and Gábor Sörös. 2013. Device recognition for intuitive interaction with the web of things. In Proceedings of the 2013 ACM conference on Pervasive and ubiquitous computing adjunct. ACM, 239-242.

[6] Ronny Seiger, Maria Gohlke, and Uwe Aßmann. 2019. Augmented Reality-Based Process Modelling for the Internet of Things with HoloFlows. In Enterprise, Business-Process and Information Systems Modeling. Springer, 115-129.

[7] Ronny Seiger, Steffen Huber, and Thomas Schlegel. 2015. Proteus: An integrated system for process execution in cyberphysical systems. In Enterprise, Business-Process and Information Systems Modeling. Springer, 265-280.

[8] Ronny Seiger, Christine Keller, Florian Niebling, and Thomas Schlegel. 2015. Modelling complex and flexible processes for smart cyber-physical environments. Journal of Computational Science 10 (2015), 137-148.

[9] Matúš Tomlein and Kaj Grønbæk. 2018. Augmented Reality Supported Modeling of Industrial Systems to Infer Software Configuration. Proceedings of the ACM on Human-Computer Interaction 2, EICS (2018), 5

[10] G. Tüysüz, B. Avenoglu, and P. E. Eren. 2013. A Workflow-Based Mobile Guidance Framework for Managing Persona Activities. In 2013 Seventh International Conference on Next Generation Mobile Apps, Services and Technologies. 13-18. 